


\title{
DiagnóSTICO DEL ESTADO DE LA COMPETENCIA DIDÁCTICA EN profesores de la Universidad Nacional del Chimborazo
}

\author{
DiAGNOSIS OF THE CONDITION O EDUCATIONAL COMPETITION IN \\ PROFESSORS OF THE NATIONAL University of Chimborazo
}

Mg. Paulo Herrera Latorre Universidad Nacional de Chimborazo/Riobamba pauloherrera@unach.edu.ec

Fecha de recepción: 15/05/2015

Fecha de aceptación: 28/06/2015

\begin{abstract}
Resumen:
A partir de desarrollar una alternativa válida para asumir el desarrollo profesional pedagógico surge el enfoque por competencias en el escenario de la educación superior, diferenciado de las concepciones que han prevalecido, al trascender el aprendizaje de contenidos teóricos por un aprendizaje demostrado en un desempeño eficaz, donde se integren conocimientos, habilidades, valores y actitudes. Las competencias de los profesionales de la educación, son aquellas que permiten solucionar los problemas inherentes al proceso pedagógico en general y al proceso de enseñanza-aprendizaje en particular. El objetivo del artículo consiste en diagnosticar la situación actual de la competencia didáctica del profesorado universitario de la Facultad de Ciencias de la Educación Humanas y Tecnologías de la Universidad Nacional de Chimborazo.
\end{abstract}




\section{Abstract:}

Based on the development of a proved alternative to asume the profesional and pedagogical improvement; appears the competences point of view at the Superior Education Level; different from those old ideas when the theoretical contents of learning emerged, throughout a demostrated learning in an ethical progress, where knowledge, abilities, values and actitudes are integrated. The competences of the professionals in education are those which allow solving problems attached to the pedagogical process in general and to the learning - teaching process in particular. The aim of this article consists on identifying the Professors` didactic competence at Facultad de Ciencias de la Educación Humanas y Tecnologías de la Universidad Nacional de Chimborazo.

Key words: Competence, Pedagogy, Education, Didactics, University. 


\section{Introducción}

El momento actual de la educación superior atraviesa un nivel del procesamiento de la información y el conocimiento nunca antes visto, los medios tecnológicos han permitido que la ciencia y su correspondiente difusión se globalicen en tiempo real. De esta forma, la educación superior debe atemperarse a las nuevas exigencias y de la manera más rápida posible realizar las trasformaciones que se requieren en la preparación de los docentes universitarios con la finalidad de dirigir un proceso formativo pertinente.

Para poder desarrollar un nivel de aprendizaje con estándares mundiales, y correspondencia con las necesidades de la realidad ecuatoriana, es necesario definir aspectos que permitan obtener información del estado actual de las competencias. En correspondencia con los aprendizajes esperados hay que dirigir el proceso de enseñanza aprendizaje, entonces ¿qué se debe tener en consideración en la dirección de ese proceso de enseñanza aprendizaje? ¿Qué competencias didácticas debe poseer el docente para logar correspondencia entre los aprendizajes y las aspiraciones curriculares?

Para formar a los docentes en las competencias didácticas que necesitan se recaba partir de cuál es su situación actual, porque ella permitirá enrumbar sobre bases concretas la labor de desarrollo de estas competencias. En la Facultad de Ciencias de la Educación, Humanas y Tecnologías de la Universidad Nacional del Chimborazo se desarrolla un proyecto de investigación que tiene como finalidad desarrollar las competencias didácticas de los docentes. El presente artículo tiene como objetivo, precisamente, exponer los resultados del estudio diagnóstico.

\section{PRESICIONES TEÓRICAS SOBRE LAS COMPETENCIAS Y LA COMPETENCIA DIDÁCTICA}

\section{Las competencias}

El enfoque por competencias proviene de la psicología cognitiva, su origen se sitúa a finales de la década del 50 del pasado siglo, cuando Noam Chomsky introduce el término de competencia lingüística. A partir de entonces ha tenido un enorme desarrollo conceptual-operacional debido a su asunción desde diversos enfoques, así como la difusión y empleo en distintos campos del conocimiento. 
Si como expresa Tobón (2010) en la década del 90 las competencias eran muy criticadas por quienes representaban los otros paradigmas educativos, con posterioridad y de manera creciente, el enfoque por competencias ha ido cobrando cada vez mayor aceptación dentro de las comunidades educativas y se encamina a convertirse en una tendencia mundial, debido a las posibilidades que brinda para movilidad estudiantil y de docentes, al reconocimiento de titulaciones por diferentes países y colocarse su valoración en el desempeño profesional. Pero además, las competencias constituyen un modo de operar en la gestión de los recursos humanos que permite una mayor articulación entre gestión, trabajo y educación; el enfoque por competencia en el mundo laboral es considerado como una herramienta que proporciona un modo de hacer y un lenguaje común.

Por estas razones los currículos de la educación superior tienden a enfocarse por competencias, como sucede en la Unión Europea con el Proyecto Tuning y en la propuesta curricular AlfaTuning para América Latina que parte de la concepción europea. No caben dudas que estas propuestas constituyen una alternativa para perfeccionar la formación de profesionales y vincular la formación con el desempeño laboral.

Dentro del estudio de las competencias profesionales se encuentran diversos enfoques, entre los que pueden mencionarse: funcionalista, conductual, constructivista y socioformativo. El principal representante de este último enfoque es el colombiano Sergio Tobón, que ha tenido mucha acogida en diversos países de Iberoamérica. Es de destacar que por lo general estos enfoques no se dan puros y es común encontrar en ellos entrelazamientos.

El enfoque socioformativo sigue los principios del pensamiento sistémico-complejo y, en esta medida, se encuentra mejor establecido para afrontar los retos actuales y futuros, caracterizados por la inter y la transdisciplinariedad, la multiplicidad de relaciones en contexto, los cambios constantes en todas las áreas y los procesos de caos e incertidumbre.

Torrado (1997) plantea que "la competencia es esencialmente un tipo de conocimiento ligado a ciertas realizaciones o desempeños, que van más allá de la memorización o la rutina. Se trata de un conocimiento derivado de un aprendizaje significativo" (p. 15). Como puede apreciarse Torrado vincula la competencia con el éxito en el desempeño profesional y la separa del conocimiento atesorado, vinculándola sólo a la significatividad del aprendizaje y saberes que se aplican.

Oscar Picardo define a las competencias como: "Una capacidad para realizar algo". Implica poseer conocimientos, habilidades, destrezas, actitudes y comportamientos armónicamente integrados para el desempeño exitoso en las distintas circunstancias de una función. No es un objeto operacional "el alumno será capaz de"..., sino: "el alumno hará tal cosa," por lo tanto sería, "saber hacer" (p. 55).

Lawshe y Balma (1966) planteaban que la competencia es: a) La potencialidad para aprender a realizar un trabajo, b) La capacidad real, actual, para llevar a cabo el trabajo, c) La disposición para realizarlo, es decir, su motivación o su interés. Estos tres aspectos se complementan, ya que es posible, que alguien tenga los conocimientos para hacer el trabajo, pero no lo desee hacer; o que tenga el deseo de realizarlo, pero no sepa cómo hacerlo; o no sepa cómo hacerlo, pero esté dispuesto a aprender y tenga las condiciones de hacerlo. 
Para Tejeda y Sánchez (2010) las competencias están integradas por un conjunto de saberes (conocimientos, habilidades, actitudes y valores) e indican que el conocimiento es el resultado de la cultura general y profesional que caracteriza al sujeto a través de conceptos, definiciones, teorías, leyes, principios, datos, informaciones, hechos, fenómenos o procesos que son aprendidos durante toda la vida.

Las competencias abarcan toda la esfera de la existencia humana y no se reducen a las competencias laborales, afectan a la personalidad en su integridad, se van adquiriendo y desarrollando a lo largo de la trayectoria profesional de los sujetos y no se pueden desvincular del contexto en el cual se genera y responden a él.

Del análisis de las definiciones anteriores la ofrecida por Tobón es la más completa, pues ella salva las insuficiencias de las demás y ofrece una visión más integral de lo que debe entender por competencias.

Sintetizando, se puede afirmar que las competencias:

- Constituyen una integración de conocimientos, habilidades, valores y actitudes.

- En su formación intervienen elementos motivacionales y metacognitivos.

- Para su desarrollo necesitan interacciones entre los sujetos, los objetos y el medio ambiente circundante, lo que tiene dimensiones tanto individuales como sociales.

- Tienen un carácter contextual.

- Se desarrollan en vínculo directo con la práctica.

- Se demuestran en el desempeño profesional, la realización exitosa de la actividad.

- Se desarrollan durante toda la vida.

\section{Competencias profesionales pedagógicas}

Las competencias profesionales pedagógicas han sido definidas y caracterizadas por diferentes autores, especialmente del contexto iberoamericano (Castellanos Simón, 2005; Braslavsky, 1999; Marcelo, 2000).

Dentro de las definiciones ofrecidas por los diferentes autores, la de Castellano Simón resulta la más completa al considerar las competencias profesionales pedagógicas como las "que permiten solucionar los problemas inherentes al proceso pedagógico en general y al proceso de enseñanza-aprendizaje en particular, en el contexto de la comunidad educativa escolar y en correspondencia con el modelo del profesional de la educación, con el propósito de promover el desarrollo integral de la personalidad de los estudiantes". (Castellanos, 2005, p. 108)

Como puede apreciarse en este concepto se destacan particularidades de la competencia profesional pedagógica:

- Permiten la solución de problemas inherentes al proceso pedagógico.

- Particularmente permiten la solución de problemas del proceso de enseñanza aprendizaje.

- Esos problemas a los cuales se les da solución están en correspondencia con el modelo del profesional (currículo). 
- La correspondencia entre las competencias y la situación contextual.

- La finalidad consiste en desarrollar integralmente la personalidad del estudiante para lo cual se necesita integrar conocimientos, habilidades, valores y actitudes.

La conceptualización ofrecida por los autores corresponde con el concepto y particularidades de competencias en general asumido anteriormente, y del que se infiere la integración de conocimientos, habilidades, valores y actitudes demostradas en un desempeño docente de calidad. Asimismo, es de destacar como estos autores precisan la particularidad, dentro de las competencias pedagógicas, de la didáctica relacionada con la dirección del proceso de enseñanza aprendizaje.

Pero cabría preguntarse ¿cuáles son las competencias pedagógicas? ¿Existe coincidencia en las clasificaciones que ofrecen los diferentes autores sobre las mismas? La realización de un breve recorrido por las clasificaciones permitirá profundizar en la naturaleza de la profesión pedagógica, al mismo tiempo apreciar a qué desempeños se les otorga mayor relieve.

Verónica Marín Díaz, de la Universidad de Huelva refiere (2004) los conocimientos Grossman (1990, citado por De Vicente, 1994) propone para el profesor universitario:

\section{Conocimiento de la materia - Conocimiento pedagógico general}

\begin{tabular}{|l|c|c|c|c|c|c|}
\hline \multicolumn{2}{|c|}{ Conocimiento de la materia } & \multicolumn{4}{c|}{ Conocimiento pedagógico general } \\
\hline $\begin{array}{c}\text { Estructuras } \\
\text { sintácticas }\end{array}$ & Contenido & $\begin{array}{c}\text { Estructuras } \\
\text { sustantivas }\end{array}$ & Aprendizaje & $\begin{array}{c}\text { Gestión de } \\
\text { clase }\end{array}$ & $\begin{array}{c}\text { Currículum e } \\
\text { instrucción }\end{array}$ & Otras \\
\hline
\end{tabular}

\section{Conocimiento didáctico del contenido}

\begin{tabular}{|c|c|c|}
\hline $\begin{array}{c}\text { Conocimiento de la } \\
\text { comprensión de los estudiantes }\end{array}$ & $\begin{array}{c}\text { Conocimiento } \\
\text { curricular }\end{array}$ & $\begin{array}{c}\text { Conocimiento de las estratégias } \\
\text { instructivas }\end{array}$ \\
\hline
\end{tabular}

\section{Conocimiento del contexto}

\begin{tabular}{|c|c|c|}
\hline Comunidad & Distrito & Escuela \\
\hline
\end{tabular}

Cuadro 1: Tipos de conocimiento. 
- Conocimiento de la materia: Dicho conocimiento está referido al conocimiento del hecho y de los conceptos más significativos tanto en el tema de estudio como en las relaciones que este permite establecer (Moral, 2000).

- Conocimiento pedagógico general: Se encuentra referido a aquel conocimiento que está relacionado directamente con el proceso de aprendizaje, el alumnado y su tiempo de aprendizaje además de aquel otro que está imbricado con el uso de las técnicas didácticas, estructuración del temario, planificación del currículum, evaluación del alumnado y del propio docente, etc.( Gudmundsdottir, 1998; Marcelo, 1993; TurnerBisset, 1999). Es, en definitiva, el que identifica a los profesionales de la educación (Sarramona; Noguera y Vera, 1998; Touriñán, 1990).

- Conocimiento didáctico del contenido: "Hace referencia a las formas de presentación del contenido de una materia asequible a los alumnos" (Moral, 1994:37). Se construye a partir del conocimiento del contenido y del pedagógico, de los alumnos y de la biografía personal y profesional del docente.

- Conocimiento del contexto: Está referido al dónde se produce la enseñanza y quién es la persona que la produce.

En síntesis, Grosman puntualiza que en el orden pedagógico el docente debe tener conocimiento para la dirección del proceso de enseñanza aprendizaje y del contexto en que se desarrolla este proceso.

Por su parte Marcelo (1999) establece los conocimientos necesarios en el orden pedagógico para el profesor universitario. El autor, al igual que Grossman, no declara competencias, pero relaciona lo que se debe dominar para desempeñarse como docente. Los conocimientos que refiere son los siguientes:

- Planificación de la enseñanza.

- Metodología didáctica.

- Motivación.

- Comunicación con los alumnos.

- Gestión de clase.

- Evaluación del alumno, del ambiente de clase y del propio

- Profesor.

- Tutorías.

- Ambiente del centro.

Cecilia Braslavsky (1999), establece cinco competencias: pedagógica-didáctica, institucional, productiva, interactiva, y especificadora.

- La competencia pedagógica-didáctica. Aunque la autora integra lo pedagógico y lo didáctico en su explicación se aprecia que alude esencialmente a lo didáctico, es decir a la dirección del proceso de enseñanza 
aprendizaje. En consecuencia se refiere al proceso de toma de decisiones, para poder atender todas las situaciones que se presentan en el proceso de enseñar y aprender; selección entre una serie de estrategias para promover los aprendizajes, el uso de las tecnología, etc.

- La institucional es la competencia. Consiste en la competencia del profesor para adaptar la macropolítica a la micropolítica de su escuela, su aula, según las necesidades y condiciones en que va a desarrollar el currículo.

- La productiva. Vinculada con el trabajo, es la competencia que permite a los profesores la vinculación con la comunidad, con el mundo laboral y social, a través de lo cual den oportunidades a los estudiantes para que intervengan como ciudadanos productivos, plantearse el problema para resolverlo en el contexto donde se desarrolla.

- La competencia interactiva. Esta competencia está relacionada con la comunicación educativa, especialmente la comunicación grupal, para lo cual han de diseñarse estrategias comunicacionales, en las que se desarrolle la interacción y el colectivismo. Persigue la comunicación entre los miembros del colectivo, de entenderse con el otro.

- La competencia especificadora. La debe tener todo profesor, pues él es graduado de una disciplina, pero por ser profesor tiene que tener los elementos pedagógicos necesarios para impartir esas clases, así como un conocimiento más amplio de las disciplinas que le permita formar al estudiante desde una visión más amplia, integral.

Siguiendo con lo planteado por Castellano Simón y otros, ellos clasifican a las competencias pedagógicas en:

- competencia didáctica,

- competencia para la orientación educativa,

- competencia para la investigación educativa,

- competencia para la Comunicación educativa y competencia para la Dirección Educacional. (Castellanos, 2005, p. 108)

Miguel Ángel Zabalza (2007) propone las siguientes competencias docentes para el profesor universitario:

Primera competencia: capacidad de planificar el proceso de enseñanza y el de aprendizaje (la capacidad de hacer los propios programas, de planificar bien la propia disciplina).

Segunda competencia: seleccionar y presentar los contenidos disciplinares.

Tercera competencia: ofrecer informaciones y explicaciones comprensibles.

La cuarta competencia: tiene que ver con la alfabetización tecnológica y el manejo didáctico de las TIC`s.

La quinta competencia: tiene que ver con gestionar las metodologías de trabajo didáctico y las tareas de aprendizaje. 
La sexta competencia: tiene que ver con relacionarse constructivamente con los alumnos.

La séptima competencia: está relacionada con las tutorías y el acompañamiento a los estudiantes.

La octava competencia: reflexionar e investigar sobre la enseñanza

La última competencia; que es un poco más cualitativa significa implicarse institucionalmente. (p. 70-90)

Finalmente, Tobón (2010) señala que las competencias docentes engloban la competencia para el trabajo en equipo, competencia para la comunicación, competencia para la planeación del proceso educativo, competencia para la evaluación del aprendizaje y competencia para la mediación del aprendizaje.

Un análisis de los conocimientos o competencias que deben poseer los profesores, expuesto por lo diferentes autores conduce a determinar cómo tendencia entre ellos que:

- La mayor significación se la otorgan a la dirección del proceso de enseñanza aprendizaje para lo cual consideran la planificación de clases, la elaboración de estrategias de aprendizaje, la evaluación, las tutorías, la comunicación, el empleo de las nuevas tecnologías, empleo de explicaciones comprensibles, lograr mayor motivación, etc.

- La relación que existe entre el modelo del profesional, el currículo, y las competencias que debe poseer el profesor, así como el contexto en que se desarrolla el proceso de formación, pues de acuerdo con este modelo y el contexto habrá que proceder pedagógicamente. De modo que "debe considerarse la relación existente entre las competencias y el modelo de desempeño socialmente establecido en cada época histórica y en cada contexto particular. Por ello, las competencias de los y las profesionales de la educación constituyen un sistema integral que permite movilizar los recursos indispensables para dirigir, orientar e investigar con eficiencia el objeto de trabajo en diferentes contextos y relaciones, atendiendo a las demandas del desarrollo social." (2005:108) Es decir, se insiste en el carácter contextual de las competencias y en su relación con el modelo del profesional, por lo que ellas no pueden ser extrapoladas indiscriminadamente.

\section{La didáctica y la competencia didáctica}

La didáctica constituye una de las ciencias pedagógicas y tiene como objeto de estudio el proceso de enseñanza aprendizaje.

Según Zilberstein Trouncha (1999). El proceso de enseñanza aprendizaje constituye la vía mediatizadora esencial para la apropiación de conocimientos, habilidades, hábitos, normas de relación, de comportamiento y valores, legados por la humanidad, que se expresan en el contenido de enseñanza, en estrecho vínculo con el resto de las actividades docentes y extradocentes que realizan los estudiantes.

El proceso de enseñanza y aprendizaje resulta una forma esencial para lograr la educación de los alumnos, pero para ello debe cumplir una serie de requerimientos y exigencias que se entienden a todos los que en él 
participan y a los diferentes elementos y componentes del propio proceso. Ambos procesos no ocurren de modo independiente, sino que forman una unidad, por, lo que no deben atenderse aisladamente, ni tampoco en relaciones bilaterales, sino constituyendo un verdadero sistema. Debe estructurarse, organizarse y orientarse en correspondencia con los requerimientos de la edad, de las condiciones y situaciones, de las particularidades individuales y del propio proceso. (Colectivo de autores, 2002, p. 45)

La brasileña V. M. Candau, (citado por González Soca, en Addine et all, 2009) deshecha la tradición mecanicista de la didáctica y propone las siguientes dimensiones:

Dimensión humana: Si bien la concepción humanista es unilateral y reduccionista, haciendo de la dimensión humana el único centro configurador del proceso de enseñanza-aprendizaje, sin embargo, ella explícita la importancia de esa dimensión. Ciertamente el componente afectivo está presente en el proceso de enseñanza-aprendizaje.

Dimensión técnica: Se refiere al proceso de enseñanza-aprendizaje como acción intencional, sistemática, que procura organizar las condiciones que mejor propician el aprendizaje. Aspectos como objetivos instruccionales, selección del contenido, estrategias de enseñanza, evaluación, etc. [...] Se trata del aspecto considerado objetivo y racional del proceso de enseñanza-aprendizaje.

Dimensión político-social: Si todo el proceso de enseñanza- aprendizaje es "situado", la dimensión político-social le es inherente. Él acontece siempre en una cultura específica, trata con personas concretas que tienen una posición de clase definida en la organización social en que viven [...] toda la práctica pedagógica [...] posee en sí una dimensión político-social.

Calos Álvarez (1999) señala que las leyes del proceso del proceso de enseñanza aprendizaje (el autor le nombra proceso docente educativo) son las siguientes:

- Ley es relaciones del proceso docente-educativo con el contexto social: La escuela en la vida, establece la relación entre la sociedad en un vínculo con el proceso docente educativo, y es lo social el que tienen el papel dirigente, en él se da el problema (la necesidad social), el objetivo (el modelo pedagógico) y el proceso, conformando así una triada dialéctica.

- Ley de las relaciones internas entre los componentes del proceso docente-educativo: La educación a través de la instrucción, se da como consecuencia de la primera ley cada unidad organizativa del proceso docente-educativo, como sistema, debe preparar al estudiante para enfrentarse a un tipo de problema y resolverlo.

Los componentes del proceso de enseñanza aprendizaje tienen los siguientes componentes:

Componente humano: profesor, estudiantes, el grupo.

Componente cultural: objetivos, contenidos, métodos, formas de organización, evaluación. (p. 70-90) 
Los distintos componentes del proceso de enseñanza aprendizaje están interrelacionados, y responde a los fundamentos del currículo y a sus exigencias, es decir, la forma de dirigir el proceso de enseñanza aprendizaje y de lograr los aprendizajes de los alumnos se encuentra prevista en el currículo y no quedan a la voluntad de los docentes. Al mismo tiempo, habrá que estimar que una cosa es el currículo formal, oficial, y otra el desarrollo curricular donde se producen situaciones, imposibles de prever en el documento, las cuales son necesario considerar para que el proceso de enseñanza fluya adecuadamente como son: las particularidades del contexto, las necesidades de los estudiantes, los profesores con sus características personológicas y preparación, los recursos y bibliografía que se posean, entre otros.

\section{Metodología para el diagnóstico}

El estudio diagnóstico de la competencia didáctica se realizó en la Facultad de Ciencias de la Educación, Humanas y Tecnologías de la Universidad Nacional de Chimborazo.

Se tuvo como punto teórico de partida el concepto de competencia didáctica anteriormente referido. Este concepto se desglosó en las siguientes dimensiones e indicadores:

\section{Diagnóstico a estudiantes}

- Si los docentes realizan diagnóstico a los estudiantes.

- Aspectos que se diagnostican.

- Técnicas que se emplean para diagnosticar.

- Procesamiento del diagnóstico.

- Diseño de acciones a partir del diagnóstico.

\section{Estrategias de enseñanza aprendizaje}

- Caracteres de las estrategias de aprendizaje que se emplean.

- El uso de las tic`s.

- La planificación de las clases atendiendo a los diferentes componentes del proceso de enseñanza aprendizaje

- La evaluación del proceso de enseñanza aprendizaje

\section{Tutoría}

- Diseño de la tutorías individuales y grupales en correspondencia con las necesidades de los estudiantes

- Resultados de las tutorías

\section{Administración del proceso de enseñanza aprendizaje}

- Elaboración de sílabos teniendo en cuenta la estructura curricular y adelantos de la ciencia desde una posición integradora.

- La planificación de las clases atendiendo al sílabo. 
Para la realización del diagnóstico se empleó la misma encuesta a profesores y estudiantes, la que permite el cruzamiento de información. La encuesta se tipifica de la siguiente manera:

1. Tipo de preguntas

2. El ambiente en que se realiza

3. Fuente de datos

$$
\begin{aligned}
& =\text { Descriptiva } \\
& =\text { Campo } \\
& =\text { Primaria }
\end{aligned}
$$

\section{Población y muestra}

La población para el diagnóstico de la investigación estuvo conformada por:

- El claustro de la Facultad de Ciencias de la Educación, Humanas y Tecnologías de la Universidad Nacional del Chimborazo que laboraba durante el primer semestre del curso 2015, conformado por 147 docentes. De la población de docentes se tomó aleatoriamente un 30\%, equivalente a 40 docentes.

- Estudiantes de los octavos semestres de la Facultad de Ciencias de la Educación, Humanas y Tecnologías de la Universidad Nacional del Chimborazo. Se escogió intencionalmente a los estudiantes del 8vo semestre, debido a su experiencia dentro de la Facultad la que permite ofrecer valoraciones más sustentadas.

\section{Resultados y Discusión}

En el análisis se sigue el orden de las preguntas formuladas en la encuesta.

\section{1. ¿Durante el proceso previo y el desarrollo de las asignaturas que imparte, diagnostica a los estudiantes?}

ANÁLISIS DOCENTES. El 90\% de los docentes expresan que diagnosticaron a los estudiantes durante el proceso previo y el desarrollo de las asignaturas que imparten, mientras que el $5 \%$ dice que no y el otro $5 \%$ dice que a veces.

ANÁLISIS ESTUDIANTES. El 57\% de los estudiantes dice que el docente diagnosticó durante el proceso previo y el desarrollo de las asignaturas, el $33 \%$ indica que lo hace a veces y el $10 \%$ indica que no lo hace. - Los resultados evidencian una gran discrepancia entre los planteado por docentes y estudiantes.

\section{Si diagnostica ¿Qué aspecto tiene en cuenta? Enumere al menos cuatro aspectos:}

ANÁLISIS DOCENTES. Los aspectos que tienen en cuenta los docentes para diagnosticar son: el 12\% la motivación del estudiante; el 10\% la actitud y la responsabilidad, ya que es importante al momento de califi- 
car; el $8 \%$ los métodos de enseñanza; el 5\% los métodos de investigación y, por último, el 3\% la estabilidad emocional y la comprensión lectora.

ANÁLISIS ESTUDIANTES. Señalan que cuando el profesor diagnostica toma en cuenta: conocimientos, el $18 \%$; la actitud, métodos de aprendizaje y capacidad, el $10 \%$; el interés por la carrera y el nivel de aprendizaje, el $8 \%$; la responsabilidad, el $7 \%$ y la relación de lo aprendido con la realidad, el $6 \%$.

\section{3. ¿Qué técnicas utiliza para diagnosticar?}

ANÁLISIS DOCENTES. El 33\% de los docentes señala que utiliza la técnica de la prueba, el 30\% utiliza el test personal, el 20\% utiliza la observación, el 13\% las encuestas, el 5\% utiliza mediante grupos de discusión, no se ocupan entrevistas.

ANÁLISIS ESTUDIANTES. El 56\% de los estudiantes señalan que los docentes utilizan las pruebas para diagnosticar, el 24\% la observación, el 10\% lo hace mediante las entrevistas, el 7\% en grupos de discusión, el 2\% mediante entrevistas y el $1 \%$ mediante test personal.

\section{4. ¿Cómo procesa los datos que le ofrece el diagnóstico?}

ANÁLISIS DOCENTES. Los docentes expresan en un $28 \%$ que procesan los datos ofrecidos por el diagnóstico mediante programas en Excel, el 20\% comparando los resultados, el 15\% mediante diagnóstico de test y tabulación de datos y el $13 \%$ mediante cuadros gráficos.

ANÁLISIS ESTUDIANTES. El 45\% de los estudiantes manifiestan que el análisis de los datos lo hacen mediante tabulación en Excel; mientras que el 13\% lo hace tabulando datos, fortaleciendo contenidos y aprendizajes y mediante lista de cotejo; el 12\% lo hace comparando los resultados de aprendizajes con los objetivos propuestos y mediante cuadros y gráficos de diagnóstico, el $5 \%$.

\section{5. ¿A partir de los resultados del diagnóstico diseña acciones para mejorar el proceso de enseñanza aprendizaje?}

ANÁLISIS DOCENTES. El 62\% de los docentes señala que utiliza los resultados del diagnóstico y diseña acciones para mejorar el proceso de enseñanza aprendizaje, eso demuestra que el docente está interesado por mejorar su enseñanza, el $25 \%$ de los docentes dice que lo hace a veces y el $13 \%$ no lo hace.

ANÁLISIS ESTUDIANTES. El 58\% de los estudiantes dice que el docente si analiza a partir de los resultados del diagnóstico, el docente diseña acciones para mejorar el proceso de enseñanza aprendizaje, el 38\% dice que lo hace a veces y el $4 \%$ no lo hace. 
6. ¿Le ofrece seguimiento a los resultados del diagnóstico durante el proceso de enseñanza aprendizaje?

ANÁLISIS DOCENTES. El 70\% de los docentes ofrece seguimiento a los resultados del diagnóstico durante el proceso de enseñanza aprendizaje, mientras el 25\% dice que a veces y el $5 \%$ no ofrece seguimiento.

ANÁLISIS ESTUDIANTES. El 49\% de los estudiantes dice que el docente ofrece seguimiento a los resultados del diagnóstico durante el proceso de enseñanza aprendizaje, el $42 \%$ dice que a veces y el $8 \%$ dice que no lo hace.

\section{7 ¿Realiza una valoración de los estudiantes y la compara con los resultados del diagnóstico inicial?}

ANÁLISIS DOCENTES. El 87\% si realiza una valoración a los estudiantes y la compara con los resultados del diagnóstico inicial, mientras que el 10\% lo hace a veces y el 3\% no lo hacen.

ANÁLISIS ESTUDIANTES. El 44\% del estudiantado dice que el docente realiza una valoración de los estudiantes y la compara con los resultados del diagnóstico inicial, mientras que el $41 \%$ dice que lo hacen a veces y el $15 \%$ no.

\section{Al elaborar los sílabos el docente tiene en cuenta:}

ANÁLISIS DOCENTES. El 35\% de los docentes antes de realizar los sílabos toma en cuenta el currículo de la carrera, mientras que el $20 \%$ toma en cuenta el desarrollo actual de la ciencia y el nivel de los alumnos, el $15 \%$ toma en cuenta los conocimientos, habilidades y valores y el 10\% toma en cuenta la interrelación con otras materias.

¿Al inicio de cada período académico el docente presenta a sus estudiantes el sílabo?

ANÁLISIS ESTUDIANTES. El 53\% de los encuestados dice que no presenta al inicio de cada período académico a sus estudiantes el sílabo, el 30\% dice que a veces y el 17\% dice que si presenta.

\section{La planificación de las clases ¿Ud. Prepara sus clases?}

ANÁLISIS DOCENTE. El 97\% de los docentes planifica sus clases con tiempo mientras que el 3\% planifica a veces.

En la elaboración de sílabos, el docente tiene en cuenta: 
ANÁLISIS ESTUDIANTES. EL 47\% de los estudiantes dice que el docente en el momento de la elaboración de los sílabos toma en cuenta el currículo de la carrera; el 23\% toma en cuenta atender a los conocimientos, habilidades y valores; el 14\% el desarrollo actual de la ciencia; el 10\% la interrelación con otras materias y el $6 \%$ el nivel de los alumnos.

\section{0. ¿Al preparar sus clases tiene en cuenta el sílabo?}

ANÁLISIS DOCENTE. El 90\% toma en cuenta los sílabos para preparar clases, mientras que el 8\% de docentes lo hace a veces y el y el $2 \%$ no toma en cuenta.

\section{¿El docente demuestra planificación de sus clases?}

ANÁLISIS ESTUDIANTES. El 43\% de los estudiantes encuestados dicen que el docente demuestra planificación de sus clases, mientras que el $41 \%$ dice que a veces y el 16\% dice que no demuestra planificación en sus clases.

\section{Si prepara clases marque con una $X$ los aspectos que Ud. tiene en cuenta:}

ANÁLISIS DOCENTE. El 24\% de los docentes encuestados dice que aplica los sílabos para preparar clases; el 20\% toma en cuenta la participación activa de los estudiantes; el 18\% la vinculación con la práctica, con la vida; el 10\% utiliza los nuevos aportes de la ciencia; el 8\% los resultados del diagnóstico de los estudiantes y usa las tics, también toma atención a las diferencias individuales y el $4 \%$ toma en cuenta el conocimientos, habilidades y valores.

¿En la preparación de clases el docente toma en cuenta el sílabo?

ANÁLISIS ESTUDIANTES. EL 60\% de los encuestados dicen que los docentes toman en cuenta los sílabos para la preparación de sus clases, el $42 \%$ dice que a veces y el $6 \%$ dicen que no toman en cuenta los docentes.

\section{Si Ud. prepara clases marque con un $X$ los aspectos que tiene en cuenta}

ANÁLISIS DOCENTE. El 30\% de los docentes hace en base a los objetivos planteados, mientras que el $20 \%$ de acuerdo a métodos de enseñanza, el $15 \%$ de acuerdo a métodos y procedimientos didácticos que empleará, el 13\% de acuerdo a contenidos, el 12\% de acuerdo a la forma de evaluación y el 10\% de los encuestados lo hace de forma de organización de la docencia (conferencia, clase práctica, seminario, práctica de laboratorio, etc.). 
Aspectos que el docente toma en cuenta en clases:

ANÁLISIS ESTUDIANTES. El 28\% de los encuestados considera que los sílabos son los aspectos que toman los docentes en el momento de impartir las clases; el 10\% utiliza los contenidos; el $8 \%$ los objetivos trazados; el 7\% medios de enseñanza, forma de organización de la docencia (conferencia, clase práctica, seminario, práctica de laboratorio, etc.), los resultados del diagnóstico de los estudiantes; el $4 \%$ considera que el docente utiliza los nuevos aportes de la ciencia, la vinculación con la práctica, con la vida y el $3 \%$ considera que la forma de evaluación, la participación activa de los estudiantes, atención a las diferencias individuales y los conocimientos, habilidades y valores.

\section{3. ¿El docente ofrece tutorías a sus estudiantes?}

ANÁLISIS DOCENTES. El 60\% de los docentes ofrece tutorías a sus estudiantes y eso es importante, ya que fortalece la enseñanza, mientras que el $5 \%$ dice que no y el $35 \%$ dice que a veces lo hace.

ANÁLISIS ESTUDIANTES. El 45\% de los estudiantes dice que el docente a veces ofrece tutorías, mientras que el $32 \%$ dice que sí y el $23 \%$ dice que no.

\section{4. ¿Las tutorías que ofrece son?}

ANÁLISIS DOCENTES. El 63\% de los docentes dice que lo lo individual o grupal, depende del momento y la ocasión; mientras que el 22\% dice que lo hace individual para mejor aprendizaje y el $15 \%$ lo hace en forma grupal.

ANÁLISIS ESTUDIANTES. Los estudiantes consideran que los docentes dan las tutorías el $43 \%$ de las dos formas tanto grupales como individuales, el $26 \%$ lo hacen grupales y el $31 \%$ lo hacen individuales.

\section{Señale tres aspectos que aborda en las tutorías}

ANÁLISIS DOCENTES. El 19\% aborda los conocimientos, el 17\% la comprensión, el 13\% hace un refuerzo de aprendizaje y las recomendaciones que le solicitan los estudiantes, el 9\% refuerza el desarrollo, el $8 \%$ se enfoca en reforzar temas comunes y las conclusiones y el 5\% toma en base al acontecer del país y el desempeño del estudiante.

ANÁLISIS ESTUDIANTES. El 16\% de los estudiantes dicen que los docentes abordan el refuerzo de aprendizaje, el 15\% refuerza temas comunes, el 14\% la comprensión, el 12\% recomendaciones, el $10 \%$ con las conclusiones, el $8 \%$ el rendimiento académico, el $7 \%$ piensa que es en base al desempeño del estudiante, el $5 \%$ de acuerdo al desarrollo y el $4 \%$ de acuerdo al acontecer del país 


\section{CONCLUSIONES}

- Con respecto al diagnóstico que realiza el docente a los estudiantes, los docentes expresan que cumplen en gran medida con este aspecto; mientras los estudiantes consideran que solo medianamente. Los parámetros que se diagnostican concuerdan ampliamente en criterio los docentes como estudiantes, lo que significa un nivel de satisfacción alto en el entorno educativo. Las técnicas empleadas para el diagnóstico son utilizadas de manera sistemática por el claustro de docentes, esto indica un seguimiento a la evaluación y los beneficios que conlleva la aplicación de la misma.

- Docentes y estudiantes concuerdan en que el proceso de evaluación o diagnóstico inicial se realiza a través de:

- Utilización de pruebas

- La observación

- Grupos de discusión

Los estudiantes agregan la aplicación de entrevistas.

- Los docentes y estudiantes concuerdan en que la planificación de las clases se da tomando en cuenta el currículo de la carrera, el desarrollo actual de la ciencia y nivel de alumnos, así como los conocimientos, habilidades y valores. Sin embargo, el porcentaje de cumplimiento de este parámetro es bajo, lo que claramente evidencia la necesidad de mejorar en este aspecto. Con respecto a la evaluación del proceso de enseñanza aprendizaje el criterio de los docentes es alto; no obstante, a decir de los estudiantes no se cumple ampliamente con este proceso. Se presenta este aspecto como oportunidad de mejora, ya que los procesos de evaluación permiten la consolidación de los conocimientos.

- La elaboración de los sílabos teniendo en cuenta la estructura curricular y adelantos de la ciencia a criterio de los docentes un bajo porcentaje indica el cumplimiento de este aspecto. Sobre la planificación de las clases atendiendo al sílabo un porcentaje mayoritario de docentes explica que si cumple, pero a criterio de los estudiantes encuestados medianamente se demuestra la planificación en este ámbito.

- Como puede observarse existe discrepancia en muchos aspectos entre lo planteado por los docentes y los estudiantes. En general, los docentes tratan de presentar una situación favorable con respecto a su competencia didáctica, mientras que los estudiantes son más críticos y evidencian debilidades en el desarrollo de la competencia didáctica de los docentes. 


\section{BIBLIOGRAFÍA}

Addine, F. (2000). Didáctica y Currículo. Bolivia: Editorial AB.

Álvarez, C. (1999). La escuela en la vida. La Habana: Editorial Pueblo y Educación.

Ansorena, C. (1996). 15 casos para la Selección de Personal con Éxito. Barcelona: Paidós Empresa.

Boekaerts, M. (1997). Self-regulated learning: a new concept embraced by researchers, policy makers, aducators, teachers, and students. Learning and Instruction, 7(2), 161-186.

Bolaños, G. y Z. Molina (2007). Introducción al curriculum. San José de Costa Rica

Boyatzis, R. (1982). The competent manager. New York: Wiley and Sons. Recuperado de: http://books.google.com.co

Braslavsky, C. (1999). Bases, orientaciones y criterios para el diseño de programas de formación de profesores. Revista Iberoamericano de Educación, (19).

Cáceres, M. (2001). La formación pedagógica de los profesores universitarios. Una propuesta necesaria. Cuba: Universidad de Cienfuegos.

Castellanos, B. \& otros. (2005). Esquema conceptual, referencial y operativo sobre la investigación educativa. La Habana: Editorial Pueblo y Educación.

Colectivo de autores (2002). Compendio de Pedagogía. La Habana: Editorial Pueblo y Educación

Fernández, B. y Valdivieso, M. (2007). El currículo y las competencias investigativas. Caracas: UPEL-IPC.

Ginés Mora, J. (2004): La necesidad del cambio educativo en la sociedad del conocimiento. Organización de Estados Iberoamericanos para la Educación, la Ciencia y la Cultura. Revista Iberoamericana de Educación. (35).

González Morales, A. (2006). La Universidad renovada. Arequipa: Editorial UNSA.

Lawshe, C.H.\& Balma, Michael J. (1966). Principles of Personnel Testing. New York: McGraw-Hill.

Marcelo, C. (1995). Formación del profesorado para el cambio educativo. Barcelona: EUB.

Marín Díaz, Verónica: (2004) Recuperado de: http://rabida.uhu.es/dspace/bitstream/handle/10272/6646/Conocimiento_y_formacion_profesorado.pdf?sequence=2

Morín, E. (2001) Los sietes saberes necesarios para la educación del futuro. Barcelona: Editorial Paidós 
Pichardo, O. (2002). Pedagogía informacional: enseñar a aprender en la sociedad del conocimiento. El Salvador: Universidad Francisco Gavidia.

UNESCO (1998). Declaración Mundial sobre la Educación Superior en el siglo XXI: Visión y Acción, y Marco de acción prioritaria para el cambio y el desarrollo de la Educación Superior. Education UNESCO. Recuperado de: http://www.education.unesco.org/educprog/wch/presentation.htm

Riera, E. (2004). Curriculum para la reconstrucción social. Revista Ciencias de la Educación, Vol. 1 (23).

Tejeda, J. y Sánchez, P. (2010). Acerca de las competencias profesionales. Revista Herramientas, (56), pp. 20-30. Recuperado de: peremarques.pangea.org/dioe/competencias.pdf)

Torrado, M. C. (1999). El desarrollo de las competencias: una propuesta para la educación colombiana. Memorias del Taller sobre Evaluación de Competencias Básicas. Bogotá: Universidad Nacional de Colombia.

Tünnermann, B. C. (2003). La universidad latinoamericana ante los retos del siglo XXI. Editorial Colección UDUAL.

Zalbalza, M. (2000). Diseño y desarrollar curricular. Madrid: Editorial Narcea.

(2007). Competencias docentes del profesor. Calidad y desarrollo profesional. España: Ediciones Narcea 


\section{ANEXOS}

ENCUESTA A DOCENTES Y ESTUDIANTES DE LA FACULTAD DE CIENCIAS DE LA EDUCACIÓN HUMANAS Y TECNOLOGÍAS.

1. ¿Durante el proceso previo y el desarrollo de las asignaturas que imparte, diagnostica a los estudiantes?

\begin{tabular}{|c|c|c|}
\hline CRITERIO DOCENTES & FRECUENCIA & PORCENTAJE \\
\hline SI & 36 & $90 \%$ \\
\hline NO & 2 & $5 \%$ \\
\hline A VECES & 2 & $5 \%$ \\
\hline TOTAL & 40 & $100 \%$ \\
\hline
\end{tabular}

\begin{tabular}{|c|c|c|}
\hline CRITERIO ESTUDIANTES & FRECUENCIA & PORCENTAJE \\
\hline SI & 83 & $57 \%$ \\
\hline NO & 14 & $10 \%$ \\
\hline A VECES & 49 & $33 \%$ \\
\hline TOTAL & 146 & $100 \%$ \\
\hline
\end{tabular}

2. Si diagnostica ¿Qué aspecto tiene en cuenta? Enumere al menos cuatro aspectos:

\begin{tabular}{|c|c|c|}
\hline CRITERIO DOCENTES & FRECUENCIA & PORCENTAJE \\
\hline Conocimientos previos & 2 & $5 \%$ \\
\hline Métodos de aprendizaje & 3 & $7 \%$ \\
\hline Motivación del aprendizaje & 5 & $12 \%$ \\
\hline Comprensión Lectora & 1 & $3 \%$ \\
\hline Responsabilidad & 4 & $10 \%$ \\
\hline Relación de lo aprendido con la \\
realidad & 2 & $5 \%$ \\
\hline Nivel de aprendizaje & 3 & $7 \%$ \\
\hline Interés por la carrera & 2 & $5 \%$ \\
\hline Rangos de aprendizaje-contenidos & 3 & $8 \%$ \\
\hline Actitud & 4 & $10 \%$ \\
\hline
\end{tabular}




\begin{tabular}{|c|c|c|}
\hline Estabilidad emocional & 1 & $3 \%$ \\
\hline Métodos de investigación & 2 & $5 \%$ \\
\hline Opinión científica & 3 & $8 \%$ \\
\hline Capacidades & 3 & $8 \%$ \\
\hline Problemas sociales & 2 & $5 \%$ \\
\hline TOTAL & $\mathbf{4 0}$ & $\mathbf{1 0 0 \%}$ \\
\hline
\end{tabular}

\begin{tabular}{|c|c|c|}
\hline CRITERIO ESTUDIANTES & FRECUENCIA & PORCENTAJE \\
\hline Conocimientos previos & 25 & $18 \%$ \\
\hline Métodos de aprendizaje & 15 & $10 \%$ \\
\hline Motivación del estudiante & 5 & $3 \%$ \\
\hline Comprensión Lectora & 4 & $3 \%$ \\
\hline Responsabilidad & 10 & $6 \%$ \\
\hline Relación de lo aprendido con la & 9 & $8 \%$ \\
\hline realidad & 11 & $8 \%$ \\
\hline Nivel de aprendizaje & 11 & $5 \%$ \\
\hline Ranterés por la carrera & 8 & $10 \%$ \\
\hline Aste aprendizaje-contenidos & 15 & $1 \%$ \\
\hline Actitud & 2 & $4 \%$ \\
\hline Métodos de investigación & 6 & $5 \%$ \\
\hline Opinión científica & 7 & $10 \%$ \\
\hline Capacidades & 15 & $2 \%$ \\
\hline Problemas sociales & 3 & $\mathbf{1 0 0 \%}$ \\
\hline TOTAL & $\mathbf{1 4 6}$ & \\
\hline & & \\
\hline & 9 & \\
\hline
\end{tabular}

\section{3. ¿Qué técnicas utiliza para diagnosticar?}

\begin{tabular}{|c|c|c|}
\hline CRITERIO DOCENTES & FRECUENCIA & PORCENTAJE \\
\hline Observación & 8 & $20 \%$ \\
\hline Encuestas & 5 & $13 \%$ \\
\hline
\end{tabular}




\begin{tabular}{|c|c|c|}
\hline Entrevistas & 0 & $0 \%$ \\
\hline Pruebas & 13 & $33 \%$ \\
\hline Test personal & 12 & $30 \%$ \\
\hline Grupos de discusión & 2 & $5 \%$ \\
\hline TOTAL & 40 & $100 \%$ \\
\hline
\end{tabular}

\begin{tabular}{|c|c|c|}
\hline CRITERIO ESTUDIANTES & FRECUENCIA & PORCENTAJE \\
\hline Observación & 35 & $24 \%$ \\
\hline Encuestas & 15 & $10 \%$ \\
\hline Entrevistas & 2 & $2 \%$ \\
\hline Pruebas & 82 & $56 \%$ \\
\hline Test personal & 2 & $1 \%$ \\
\hline Grupos de discusión & 10 & $7 \%$ \\
\hline TOTAL & 40 & $100 \%$ \\
\hline
\end{tabular}

\section{4. ¿Cómo procesa los datos que le ofrece el diagnóstico?}

\begin{tabular}{|l|c|c|}
\hline \multicolumn{1}{|c|}{ CRITERIO DOCENTES } & FRECUENCIA & PORCENTAJE \\
\hline Tabulando en Excel & 11 & $28 \%$ \\
\hline Tabulando los datos, fortaleciendo contenidos y aprendizajes. & 6 & $15 \%$ \\
\hline Comparando los resultados de aprendizajes con los objetivos propuestos & 8 & $20 \%$ \\
\hline $\begin{array}{l}\text { Cuadros y gráficos } \\
\text { Diagnósticos tests de métodos de estudio }\end{array}$ & 5 & $13 \%$ \\
\hline Diagnósticos tests de métodos de estudio & 6 & $15 \%$ \\
\hline Mediante lista de cotejo & 4 & $10 \%$ \\
\hline \multicolumn{1}{|c|}{ TOTAL } & 40 & $100 \%$ \\
\hline
\end{tabular}

\begin{tabular}{|l|c|c|}
\hline \multicolumn{1}{|c|}{ CRITERIO ESTUDIANTES } & FRECUENCIA & PORCENTAJE \\
\hline Tabulando en Excel & 65 & $45 \%$ \\
\hline Tabulando los datos, fortaleciendo contenidos y aprendizajes. & 19 & $13 \%$ \\
\hline Comparando los resultados de aprendizajes con los objetivos propuestos & 17 & $12 \%$ \\
\hline
\end{tabular}




\begin{tabular}{|l|c|c|}
\hline $\begin{array}{l}\text { Cuadros y gráficos } \\
\text { Diagnósticos tests de métodos de estudio }\end{array}$ & 17 & $12 \%$ \\
\hline Diagnósticos tests de métodos de estudio & 8 & $5 \%$ \\
\hline Mediante lista de cotejo & 20 & $13 \%$ \\
\hline TOTAL & 146 & $100 \%$ \\
\hline
\end{tabular}

5. ¿A partir de los resultados del diagnóstico diseña acciones para mejorar el proceso de enseñanza aprendizaje?

\begin{tabular}{|c|c|c|}
\hline CRITERIO DOCENTES & FRECUENCIA & PORCENTAJE \\
\hline SI & 25 & $62 \%$ \\
\hline NO & 5 & $13 \%$ \\
\hline A VECES & 10 & $25 \%$ \\
\hline TOTAL & 40 & $100 \%$ \\
\hline
\end{tabular}

\begin{tabular}{|c|c|c|}
\hline CRITERIO ESTUDIANTES & FRECUENCIA & PORCENTAJE \\
\hline SI & 85 & $58 \%$ \\
\hline NO & 5 & $4 \%$ \\
\hline A VECES & 56 & $38 \%$ \\
\hline TOTAL & 146 & $100 \%$ \\
\hline
\end{tabular}

6. ¿Le ofrece seguimiento a los resultados del diagnóstico durante el proceso de enseñanza aprendizaje?

\begin{tabular}{|c|c|c|}
\hline CRITERIO DOCENTES & FRECUENCIA & PORCENTAJE \\
\hline SI & 28 & $70 \%$ \\
\hline NO & 2 & $5 \%$ \\
\hline A VECES & 10 & $25 \%$ \\
\hline TOTAL & 40 & $100 \%$ \\
\hline
\end{tabular}

\begin{tabular}{|c|c|c|}
\hline CRITERIO ESTUDIANTES & FRECUENCIA & PORCENTAJE \\
\hline SI & 72 & $49 \%$ \\
\hline NO & 11 & $8 \%$ \\
\hline A VECES & 63 & $43 \%$ \\
\hline TOTAL & 146 & $100 \%$ \\
\hline
\end{tabular}


7. ¿Realiza una valoración de los estudiantes y la compara con los resultados del diagnóstico inicial?

\begin{tabular}{|c|c|c|}
\hline CRITERIO DOCENTES & FRECUENCIA & PORCENTAJE \\
\hline SI & 35 & $87 \%$ \\
\hline NO & 1 & $3 \%$ \\
\hline A VECES & 4 & $10 \%$ \\
\hline TOTAL & 40 & $100 \%$ \\
\hline
\end{tabular}

\begin{tabular}{|c|c|c|}
\hline CRITERIO ESTUDIANTES & FRECUENCIA & PORCENTAJE \\
\hline SI & 64 & $44 \%$ \\
\hline NO & 22 & $15 \%$ \\
\hline A VECES & 60 & $41 \%$ \\
\hline TOTAL & 146 & $100 \%$ \\
\hline
\end{tabular}

8. Al elaborar los sílabos el docente tiene en cuenta:

\begin{tabular}{|l|c|c|}
\hline \multicolumn{1}{|c|}{ CRITERIO DOCENTES } & FRECUENCIA & PORCENTAJE \\
\hline El currículo de la carrera & 14 & $35 \%$ \\
\hline El desarrollo actual de la ciencia & 8 & $20 \%$ \\
\hline Atender a los conocimientos, habilidades y valores & 6 & $15 \%$ \\
\hline La interrrelación con otras materias & 4 & $10 \%$ \\
\hline El nivel de los alumnos & 8 & $20 \%$ \\
\hline \multicolumn{1}{|c|}{ TOTAL } & 40 & $100 \%$ \\
\hline
\end{tabular}

Al inicio de cada período académico el docente presenta a sus estudiantes el sílabo?

\begin{tabular}{|c|c|c|}
\hline CRITERIO ESTUDIANTES & FRECUENCIA & PORCENTAJE \\
\hline SI & 25 & $17 \%$ \\
\hline NO & 78 & $53 \%$ \\
\hline A VECES & 43 & $30 \%$ \\
\hline TOTAL & 146 & $100 \%$ \\
\hline
\end{tabular}


9. La planificación de las clases Ud. Prepara sus clases?

\begin{tabular}{|c|c|c|}
\hline CRITERIO DOCENTES & FRECUENCIA & PORCENTAJE \\
\hline SI & 39 & $97 \%$ \\
\hline NO & 0 & $0 \%$ \\
\hline A VECES & 1 & $3 \%$ \\
\hline TOTAL & 40 & $100 \%$ \\
\hline
\end{tabular}

En la elaboración de sílabos, el docente tiene en cuenta:

\begin{tabular}{|l|c|c|}
\hline \multicolumn{1}{|c|}{ CRITERIO ESTUDIANTES } & FRECUENCIA & PORCENTAJE \\
\hline El currículo de la carrera & 69 & $47 \%$ \\
\hline El desarrollo actual de la ciencia & 21 & $14 \%$ \\
\hline Atender a los conocimientos, habilidades y valores & 33 & $23 \%$ \\
\hline La interrrelación con otras materias & 15 & $10 \%$ \\
\hline El nivel de los alumnos & 8 & $6 \%$ \\
\hline \multicolumn{1}{|c|}{ TOTAL } & 146 & $100 \%$ \\
\hline
\end{tabular}

10. ¿Al preparar sus clases tiene en cuenta el sílabo?

\begin{tabular}{|c|c|c|}
\hline CRITERIO DOCENTES & FRECUENCIA & PORCENTAJE \\
\hline SI & 36 & $90 \%$ \\
\hline NO & 1 & $2 \%$ \\
\hline A VECES & 3 & $8 \%$ \\
\hline TOTAL & 40 & $100 \%$ \\
\hline
\end{tabular}

¿El docente demuestra planificación de sus clases?

\begin{tabular}{|c|c|c|}
\hline CRITERIO ESTUDIANTES & FRECUENCIA & PORCENTAJE \\
\hline SI & 63 & $43 \%$ \\
\hline NO & 23 & $16 \%$ \\
\hline A VECES & 60 & $41 \%$ \\
\hline TOTAL & 146 & $100 \%$ \\
\hline
\end{tabular}


11. Si prepara clases marque con una $X$ los aspectos que Ud. tiene en cuenta:

\begin{tabular}{|c|c|c|}
\hline CRITERIO DOCENTES & FRECUENCIA & PORCENTAJE \\
\hline El sílabo & 10 & $24 \%$ \\
\hline Conocimientos, habilidades y valores & 2 & $4 \%$ \\
\hline Los nuevos aportes de la ciencia & 4 & $10 \%$ \\
\hline Los resultados del disgnóstico de los estudiantes & 3 & $8 \%$ \\
\hline Atención a las diferencias individuales & 3 & $8 \%$ \\
\hline La participación activa de los estudiantes & 8 & $20 \%$ \\
\hline Vinculación con la práctica, con la vida & 7 & $18 \%$ \\
\hline Uso de las TICs & 3 & $8 \%$ \\
\hline TOTAL & 40 & $100 \%$ \\
\hline
\end{tabular}

En la preparación de clases el docente toma en cuenta el sílabo?

\begin{tabular}{|c|c|c|}
\hline CRITERIO ESTUDIANTES & FRECUENCIA & PORCENTAJE \\
\hline SI & 88 & $60 \%$ \\
\hline NO & 9 & $6 \%$ \\
\hline A VECES & $49 \%$ & $34 \%$ \\
\hline TOTAL & 146 & $100 \%$ \\
\hline
\end{tabular}

12. Si Ud. prepara clases marque con un $X$ los aspectos que tiene en cuenta

\begin{tabular}{|c|c|c|}
\hline CRITERIO DOCENTES & FRECUENCIA & PORCENTAJE \\
\hline Objetivos & 12 & $30 \%$ \\
\hline Contenidos & 5 & $13 \%$ \\
\hline Métodos y procedimientos didácticos que empleará & 6 & $15 \%$ \\
\hline $\begin{array}{l}\text { Forma de organización de la docencia (conferencia, clase, } \\
\text { práctica, seminario, práctica de laboratorio, etc.) }\end{array}$ & 4 & $10 \%$ \\
\hline Medios de enseñanza & 8 & $20 \%$ \\
\hline Forma de evaluación & 5 & $12 \%$ \\
\hline TOTAL & 40 & $100 \%$ \\
\hline
\end{tabular}




\section{Aspectos que el docente toma en cuenta en clases:}

\begin{tabular}{|l|c|c|}
\hline \multicolumn{1}{|c|}{ CRITERIO ESTUDIANTES } & FRECUENCIA & PORCENTAJE \\
\hline El sílabo & 41 & $28 \%$ \\
\hline Conocimientos, habilidades y valores & 5 & $3 \%$ \\
\hline Los nuevos aportes de la ciencia & 6 & $4 \%$ \\
\hline Los resultados del disgnóstico de los estudiantes & 10 & $7 \%$ \\
\hline Atención a las diferencias individuales & 5 & $3 \%$ \\
\hline La participación activa de los estudiantes & 4 & $3 \%$ \\
\hline Vinculación con la práctica, con la vida & 6 & $4 \%$ \\
\hline Uso de las TICs & 10 & $7 \%$ \\
\hline Objetivos & 12 & $8 \%$ \\
\hline Contenidos & 15 & $10 \%$ \\
\hline Métodos y procedimientos didácticos que empleará & 8 & $6 \%$ \\
\hline $\begin{array}{l}\text { Forma de organización de la docencia (conferencia, clase, } \\
\text { práctica, seminario, práctica de laboratorio, etc.) }\end{array}$ & 10 & $7 \%$ \\
\hline Medios de enseñanza & 9 & $7 \%$ \\
\hline Forma de evaluación & 5 & $3 \%$ \\
\hline & 146 & $100 \%$ \\
\hline
\end{tabular}

\section{3. ¿El docente ofrece tutorías a sus estudiantes?}

\begin{tabular}{|c|c|c|}
\hline CRITERIO DOCENTES & FRECUENCIA & PORCENTAJE \\
\hline SI & 24 & $60 \%$ \\
\hline NO & 2 & $5 \%$ \\
\hline A VECES & 14 & $35 \%$ \\
\hline TOTAL & 40 & $100 \%$ \\
\hline
\end{tabular}

\begin{tabular}{|c|c|c|}
\hline CRITERIO ESTUDIANTES & FRECUENCIA & PORCENTAJE \\
\hline SI & 47 & $32 \%$ \\
\hline NO & 34 & $23 \%$ \\
\hline A VECES & 14 & $35 \%$ \\
\hline TOTAL & 146 & $100 \%$ \\
\hline
\end{tabular}


14. ¿Las tutorías que ofrece son?

\begin{tabular}{|c|c|c|}
\hline CRITERIO DOCENTES & FRECUENCIA & PORCENTAJE \\
\hline Individuales & 9 & $22 \%$ \\
\hline Grupales & 6 & $15 \%$ \\
\hline Ambas & 25 & $63 \%$ \\
\hline TOTAL & 40 & $100 \%$ \\
\hline
\end{tabular}

\begin{tabular}{|c|c|c|}
\hline CRITERIO ESTUDIANTES & FRECUENCIA & PORCENTAJE \\
\hline Individuales & 45 & $31 \%$ \\
\hline Grupales & 38 & $26 \%$ \\
\hline Ambas & 63 & $43 \%$ \\
\hline TOTAL & 146 & $100 \%$ \\
\hline
\end{tabular}

15. Señale tres aspectos que aborda en las tutorías

\begin{tabular}{|c|c|c|}
\hline CRITERIO DOCENTES & FRECUENCIA & PORCENTAJE \\
\hline Desempeño del estudiante & 2 & $5 \%$ \\
\hline Desarrollo & 4 & $9 \%$ \\
\hline Rendimiento académico & 1 & $3 \%$ \\
\hline Conclusiones & 3 & $8 \%$ \\
\hline Recomendaciones & 5 & $13 \%$ \\
\hline Comprensión & 7 & $17 \%$ \\
\hline Refuerzo del aprendizaje & 5 & $13 \%$ \\
\hline Reforzar temas comunes & 3 & $8 \%$ \\
\hline Acontecer del país & 2 & $5 \%$ \\
\hline TOTAL & $\mathbf{4 0}$ & $\mathbf{1 0 0 \%}$ \\
\hline
\end{tabular}

\begin{tabular}{|c|c|c|}
\hline CRITERIO ESTUDIANTES & FRECUENCIA & PORCENTAJE \\
\hline Desempeño del estudiante & 10 & $7 \%$ \\
\hline Desarrollo & 8 & $5 \%$ \\
\hline
\end{tabular}


Revista Científica UISRAEL, Volumen No 2; 2015 - 3, ISSN 1390-8545

\begin{tabular}{|c|c|c|}
\hline Rendimiento académico & 12 & $8 \%$ \\
\hline Conclusiones & 15 & $10 \%$ \\
\hline Recomendaciones & 17 & $12 \%$ \\
\hline Comprensión & 20 & $14 \%$ \\
\hline Refuerzo del aprendizaje & 23 & $16 \%$ \\
\hline Reforzar temas comunes & 22 & $15 \%$ \\
\hline Acontecer del país & 6 & $4 \%$ \\
\hline TOTAL & $\mathbf{1 4 6}$ & $\mathbf{1 0 0 \%}$ \\
\hline
\end{tabular}

